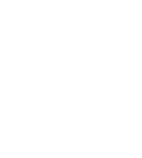
ANNUAL Further
REVIEWS

Click here for quick links to Annual Reviews content online, including:

- Other articles in this volume

- Top cited articles

- Top downloaded articles

- Our comprehensive search

Annu. Rev. Condens. Matter Phys. 2015. 6:155-75

The Annual Review of Condensed Matter Physics is online at conmatphys.annualreviews.org

This article's doi:

10.1146/annurev-conmatphys-031214-014542

Copyright $(9) 2015$ by Annual Reviews.

All rights reserved

\section{Exciton-Polariton Bose-Einstein Condensates}

\section{Benoît Deveaud}

Institute of Condensed Matter Physics, École Polytechnique Fédérale de Lausanne (EPFL), 1015 Lausanne Switzerland; email: benoit.deveaud@epfl.ch

\section{Keywords}

exciton, polariton, Bose-Einstein condensation, superfluid, vortex, Bogoliubov transformation

\begin{abstract}
Exciton-polaritons, mixed light-matter quasiparticles in semiconductors, have recently shown evidence for Bose-Einstein condensation. Some of the properties of condensates of exciton-polaritons are reviewed in this article. We first discuss the spontaneous appearance of long-range order and the way this can be easily accessed in the case of polariton fluids. We show that the Penrose-Onsager criterion is valid even for such a very special case of condensate. We then describe the experiments that allow observation of topological defects in the fluid: quantized vortices, half vortices, and hyperbolic spin vortices. We demonstrate through the comparison with the gross Pitaevskii equation that the appearance and stability of such vortices are linked with the dissipative nature of the condensate together with the presence of disorder. We then briefly summarize the experiments on superfluid behavior of the polaritons at largeenough densities and expand somewhat more on the dynamical behavior of turbulence in the wake of an obstacle, with the appearance of vortex streets. We finally show that the Bogoliubov transformation has been revealed through four-wave mixing experiments.
\end{abstract}




\section{INTRODUCTION}

Bose-Einstein condensation of quasiparticles in solids was proposed fifty years ago as a promising solution for the observation of quantum fluid effects at reasonable temperatures. Different quasiparticles have been considered with this aim, the first being excitons, as they are ideal candidates $(1,2)$. Excitons are indeed bosonic particles with a very light mass, which should allow observation of condensation effects at much higher temperatures than what is requested for atom condensates $(3,4)$. The idea to observe Bose-Einstein condensation with excitons has been pursued further by Keldysh (5), who introduced the now widely studied possibility of a transition between a Bose-Einstein condensate (BEC) and Bardeen-Cooper-Schrieffer (BCS) pairing for excitons.

The major advantage of using quasiparticles in solids corresponds to the possibility of finding a phase transition and quantum fluid effects at much higher temperatures than what must be achieved for atom vapors. At the same time, the use of solid-state-based structures allows major advantages because of the possibilities offered by modern techniques such as nanotechnology. Such advantages may play a major role for future novel devices; they also allow very interesting options for the study of the basic properties of polariton fluids, such as, for example, the creation of one-dimensional (1D) channels for polariton condensates (6), or of even more complex structures, such as, for example, Mach-Zehnder interferometers (7).

Exciton condensation in semiconductors has been the subject of many papers since the original proposals by Blatt and Moskalenko (for example, see 8, 9, 10). It is not the purpose of the present review to expand on this part of the literature.

Exciton-polaritons in semiconductor microcavities are composite particles made through the strong coupling of photons and excitons. The photon part of the state comes from photons that are confined in a semiconductor microcavity, similar to the ones that are used for the realization of vertical surface-emitting lasers (VCSELs). The exciton part of the state comes from excitons that are confined in quantum wells placed at the antinodes of the cavity modes in the microcavity.

VCSELs are very widely used for many reasons, in particular because of their very-lowthreshold current density (11). The principle of VCSELs has been exposed in great detail in the seminal paper by Jack Jewel (12). It can be summarized in the very simplified scheme of Figure 1.

Quantum wells can be introduced within the spacer layer of the cavity, ensuring that the resonance energy of the excitons matches the photon mode. This matching may be performed by adjusting the width and composition of the quantum well as well as the thickness of the spacer layer. One or up to more than 20 quantum wells can be included, provided they are positioned at the antinodes of the electromagnetic field in the cavity. For our purpose here, the main point is that photons can be confined in the cavity for a sufficiently long time, this time being measured by the $Q$ factor of the cavity. The energy of the photons confined in the cavity is given by (through a firstorder expansion):

$$
E_{p b}\left(k_{x y}\right)=\frac{\hbar c}{n_{c}} k_{z}+\frac{1}{2} \frac{\hbar c}{n_{c}} \frac{k_{x y}^{2}}{k_{z}} .
$$

This photon dispersion describes a photon that is able to propagate in the plane between the two mirrors with a mass roughly four orders of magnitude smaller than the electron rest mass.

In the same way, excitons confined in quantum wells are also able to move in the plane of the quantum well, with a mass that is approximately equal to the mass of the electron: 
a
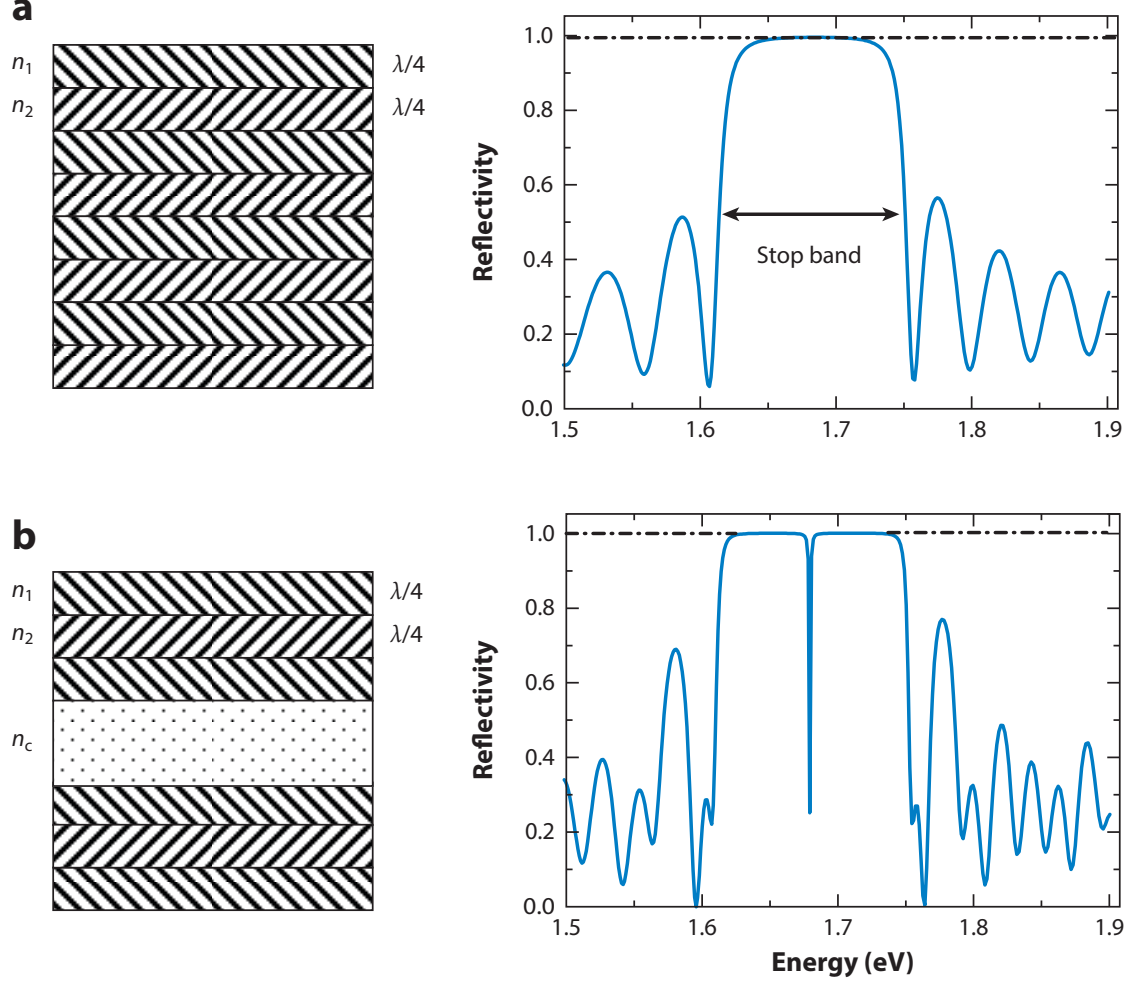

Figure 1

Modern semiconductor growth technologies allow the pile-up of $\lambda / 4$ layers of different semiconductor layers, with slightly different indexes. (a) This allows production of Bragg mirrors with very high reflectivity. $(b)$ Separating two Bragg mirrors by a spacer layer that has a thickness of $\lambda / 2$ or multiples of $\lambda / 2$ creates one photon mode confined in the system. When this photon mode is resonant with a gain medium, it produces a very efficient laser, the so-called vertical surface-emitting laser (VCSEL). Figure adapted from Reference 13.

$$
E_{x}\left(k_{x y}\right)=E_{x}(0)-\frac{\hbar^{2} k_{x y}^{2}}{2 M_{x}} .
$$

When the quality of the mirrors is high enough, this allows us to obtain the so-called strong coupling regime, where excitons and photons build up new quasiparticles called polaritons. In simplified terms, the dispersion of polaritons is

$$
E_{L P}=\frac{E_{x}\left(k_{x y}\right)+E_{p h}\left(k_{x y}\right)}{2}-\frac{1}{2} \sqrt{\left(E_{p h}\left(k_{x y}\right)-E_{x}\left(k_{x y}\right)\right)^{2}+4 \hbar^{2} \Omega^{2}}
$$

and

$$
E_{U P}=\frac{E_{x}\left(k_{x y}\right)+E_{p h}\left(k_{x y}\right)}{2}+\frac{1}{2} \sqrt{\left(E_{p h}\left(k_{x y}\right)-E_{x}\left(k_{x y}\right)\right)^{2}+4 \hbar^{2} \Omega^{2}},
$$

with $\Omega$ being the strength of the exciton-photon coupling, often referred to as the Rabi energy, which depends on material parameters for the quantum well as well as on the number of quantum wells in the cavity. In the above set of equations, the two modes of the polaritons appear: the lower 
polariton (LP), i.e., the lowest energy state of the system, and the upper polariton (UP). Both branches are approximately parabolic around $\mathrm{k}=0$ and possess an exciton (resp. photon) fraction that depends on the detuning between the uncoupled exciton and photon states. The exciton and photon fractions correspond to the respective coefficients in the wavefunction of polaritons.

Polaritons are made of photons and excitons and show properties that originate from the properties of the constituents. From the photon, they get a very small effective mass, and from the exciton, they receive the ability to interact amongst themselves because of the fermionic nature of the electron and the hole bound together in the exciton. From the photon, they receive a lifetime that is limited by the quality factor of the microcavity, i.e., how long the photons are trapped in between the two mirrors. Even if recent reports seem to indicate lifetimes in excess of $100 \mathrm{ps}$ (14), more than two orders of magnitude larger than the typical interaction time between two polaritons or between polaritons and the phonon bath of the semiconductor structure, most of the published results make use of microcavities with photon lifetimes between 1 and 10 ps.

This limited lifetime also comes as an advantage for polaritons: When leaving the microcavity, as sketched in Figure 2, the photon portion of the polariton carries with it all relevant information on the polariton population in the cavity. From the angle of the emission, one finds the wavevector of polaritons in the cavity:

$$
\hbar^{2} c^{2} k_{z}^{2}=E_{p}^{2}-\hbar^{2} c^{2} k_{\|}^{2}, \text { which leads to } \sin \theta=k_{\|} \frac{\hbar c}{E_{p}} .
$$

From the intensity at one given angle, one finds the occupancy of the polaritons at a given k. Similarly, the coherence, noise, and polarization properties are conveyed by the emitted photons. The direct correlation of such properties has been theoretically studied in detail (for example, see 16, 17). It allows, as has been done, for example, in the PhD work of J. Kasprzak (13), plotting of the polariton dispersion and occupation factors in a very direct way. Similarly, it allows obtainment, from the detected light, of the second-order coherence properties of polaritons $(18,19)$.
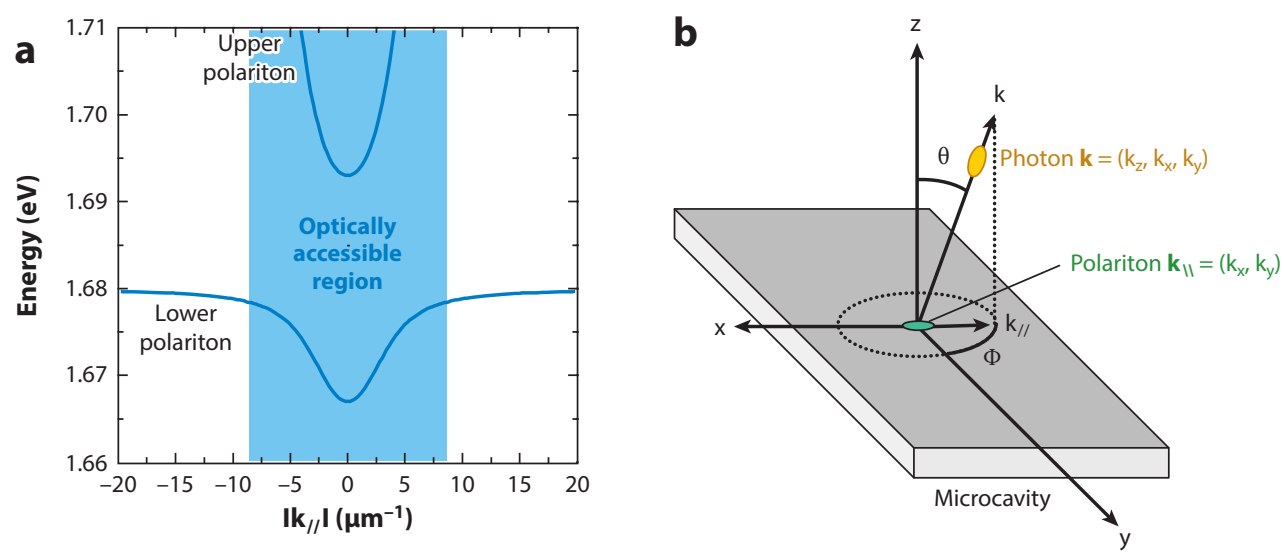

Figure 2

(a) Polariton dispersion (upper and lower branches), shown as a blue area, is the region in which the polaritons may emit light. This region basically corresponds to the energy range in which the wavevector of the polaritons can be matched by the momentum of the outgoing photon. This is very similar to refraction when exiting a dielectric material. $(b)$ Detailed matching of the in-plane polariton momentum to the emitted photon momentum. Figure adapted from Reference 15. 


\section{CONDENSATION IN A DRIVEN DISSIPATIVE SYSTEM}

Because of this very short lifetime of polaritons, an outstanding question has been raised for quite some time about the possibility to observe a phase transition, as is observed in the case of cold atom condensates. The initial demonstration of the condensation in the lower branch of polaritons (20) follows after preliminary measurements made in different groups, and, in particular, those by the group of Dang in Grenoble, where the first highly nonlinear luminescence emission by polaritons has been observed (21; see also Reference 22 for information on GaAs-based cavities). The lower polariton condensation, initially demonstrated in CdTe-based microcavities, has been confirmed in many different semiconductor systems (for example, see 23, 24) and even up to room temperature in $\mathrm{GaN}$ (25), $\mathrm{ZnO}$ (26), and organic semiconducting cavities $(27,28)$.

The assessment of the Bose-Einstein condensation, under nonresonant excitation of the system, relies on many different observations, which are described in the paper by Kasprzak and coworkers (20), such as a nonlinear threshold with the piling of polaritons at $\mathrm{k}=0$, which is a clear indication of a Bose distribution with vanishing chemical potential and an increase of temporal coherence together with a decrease of the line width. The decisive observation was the measurement of long-range spatial order through interferometric studies with the experimental system sketched in Figure 3. Figure 3 shows the first version of the interferometer, where the two images of the condensate are simply displaced from one another. In the subsequent versions of the system, one image of the condensate is interfered with by the centrosymmetric mirror image of the same condensate, allowing the probing of a long-range coherent over the entire spot at once. The ability to carry out, in a very direct way, interferometric measurements on the polariton population is a major advantage of polaritons and has served as a basis for many different observations. Some of which are described in the present review.

Without rephrasing in detail what has been described in Reference 18, let us just emphasize the fact that, below threshold, the coherence of the polariton cloud typically extends over 2 microns, approximately the de Broglie wavelength of polaritons, at the measured temperature of $19 \mathrm{~K}$. Above the condensation threshold, correlations are observed over most of the excitation spot, up to distances between correlated polaritons of more than $20 \mu \mathrm{m}$.

An estimation of the density of polaritons per quantum well at threshold has been performed by Jacek Kasprzak in his PhD thesis (13). The threshold occurs for densities on the order of $10^{10} \mathrm{~cm}^{-2}$, i.e., 50 times below the saturation density computed for the dense packing of excitons. Similar threshold densities have since been obtained in a number of other semiconductor material systems. As one example, the review paper of Bajoni (29) details the conditions of polariton condensation versus standard VCSEL lasing. The densities differ by a factor of approximately 50 .

Since our initial observation, ample literature on the subject has allowed us to understand in greater detail the behavior of exciton-polaritons and the phase transition that occurs in microcavities. The number of theoretical papers on this issue does not allow a complete overview here, and I provide only a partial reference to some of these works.

The initial idea that polariton in microcavities could undergo Bose-Einstein condensation comes from the seminal work of Imamoglu and coworkers (30), who considered the fact that a polariton condensate would behave, from the point of view of light emission, as an inversionless laser. They also considered the importance of an exciton-like polariton reservoir and detailed the possible transition from a polariton laser toward a standard laser (a VCSEL).

In order to describe as completely as possible the behavior of a polariton condensate and of its dynamics, pumping and decay of the polaritons must be included. This has been performed, for example, by Szymanska et al. (31), who showed that the appearance of coherence in a polariton condensate differs from what would occur in a VCSEL and occurs at lower densities. The standard methodology that allows a simple but clear understanding of the system, at the mean-field level, is 


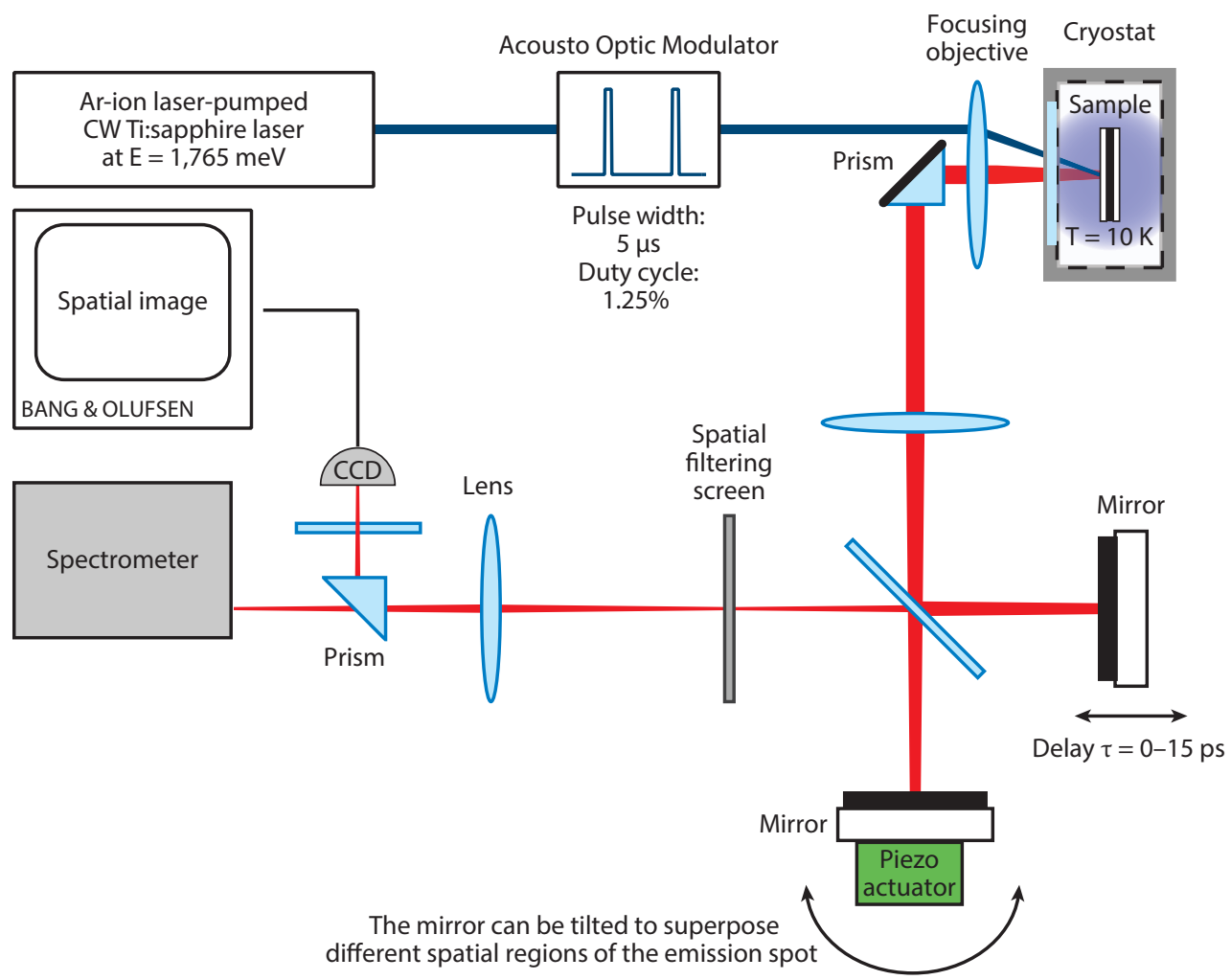

Figure 3

Experimental setup used for the measurement of the long-range coherence of polariton condensates. The microcavity is excited at high energy above the stop-band of the cavity. The whole system is then efficiently excited and relaxes to the lower polariton branch. The polariton emission is then sent to a Michelson interferometer. The stability of the interferometer is actively locked to more than $\lambda / 20$ by a HeNe laser. Figure taken from Reference 49. Abbreviations: CCD, charge-coupled device; CW, continuous wave.

the generalized Gross-Pitaevskii equation (GGPE) (or the Ginzburg-Landau equation) (for example, see 32, 33).

Very generally, the lower polariton branch is represented in a simplified way through a parabolic dispersion, and the pumping of the condensate can be provided either resonantly by the laser or nonresonantly through the presence of a reservoir [which represents the excitations of the system that do not relax immediately to the lower polariton branch: the so-called polariton bottleneck (34)]. The upper polariton branch, which is at higher energy than the reservoir, does not need to be considered in this equation because it is not appreciably populated. The equations appear as follows: a GGPE describes the macroscopic wavefunction $\psi$ of the condensate, with gain and loss terms:

$i \hbar \frac{\partial \psi(\boldsymbol{r}, t)}{\partial t}=\left\{-\frac{\hbar^{2} \nabla^{2}}{2 m_{L P}}+V_{d}(\boldsymbol{r}, t)+\frac{i \hbar}{2}\left[R\left(n_{R}(\boldsymbol{r}, t)\right)-\gamma\right]+\hbar g|\psi(\boldsymbol{r}, t)|^{2}+2 \hbar g n_{R}(\boldsymbol{r}, t)\right\} \psi(\boldsymbol{r}, t)$.

The loss term $\gamma$ corresponds mainly to the leakage of the photon portion of the polariton through the mirrors of the microcavity. The gain term, in the present case where nonresonant pumping is 
assumed, tries to model in the simplest possible way the stimulated scattering from the large $\mathrm{k}$ excitons that do not directly couple to the cavity modes on the lower polariton branch. The noncondensed fraction of the condensate does not appear in this equation because of its small influence on the evolution of the condensed fraction. The term $V_{d}(\boldsymbol{r})$ describes the effect of the unavoidable disorder in the sample [mainly caused by the fluctuations in the DBR (distributed Bragg reflector) mirrors]. In the simplest possible version, the density $n_{R}(\boldsymbol{r})$ of the reservoir is modeled by the following equation:

$$
\frac{\partial n_{R}(\boldsymbol{r}, t)}{\partial t}=P_{R}(\boldsymbol{r}, t)-\gamma_{R} n_{R}(\boldsymbol{r}, t)-R\left(n_{R}\right)|\psi(\boldsymbol{r}, t)|^{2}+\frac{1}{\tau_{I}} n_{I}(\boldsymbol{r}, t)+D \nabla^{2} n_{R}(\boldsymbol{r}, t),
$$

where the pump laser induces the first term in the right-hand side, and the last term represents the possible diffusion of excitons in the reservoir. Such diffusion effects can be neglected in most cases. The last term is included only when a second reservoir is included in time-resolved experiments (see below). Such a simple model allows reproduction of the main features of the Bose-Einstein condensation of polaritons under continuous wave $(\mathrm{CW})$ pumping.

Figure 4 shows the results of the solution of such equations (GGPE + reservoir) in the presence of a disorder that mimics what exists in the CdTe cavity that we have studied (35). This particular realization of a random disorder allows obtainment of the pinning of a quantized vortex at one of the minima of the potential disorder.

The arrows in the left part of Figure 4 indicate the direction of the flow of polaritons in the condensate. It is quite interesting to note that the density of the reservoir is almost depleted because of stimulated scattering to the condensate, except at the position of the vortex, where the density of the condensate vanishes, and therefore stimulated scattering also vanishes.

The phase of the condensate is computed in Figure 5 for exactly the same conditions as for Figure 4. It is easy to observe the phase structure that is linked with the pinned vortex and the clear

a

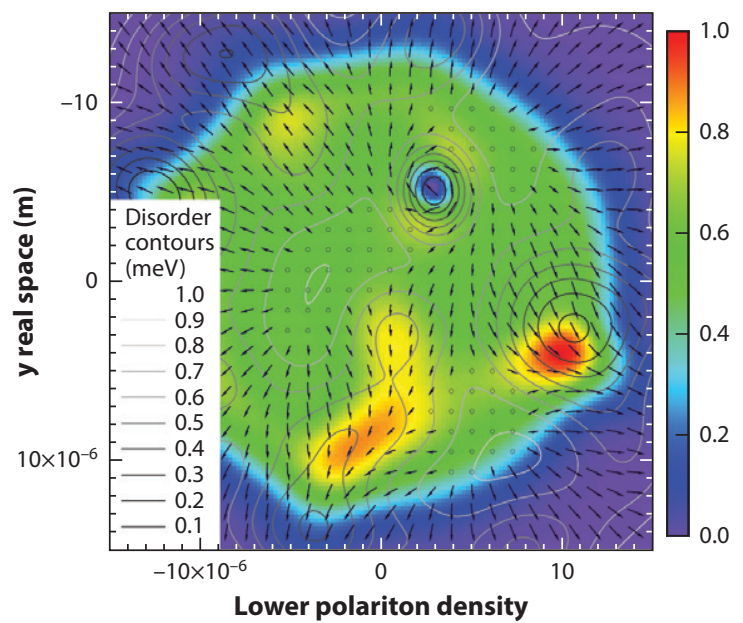

b

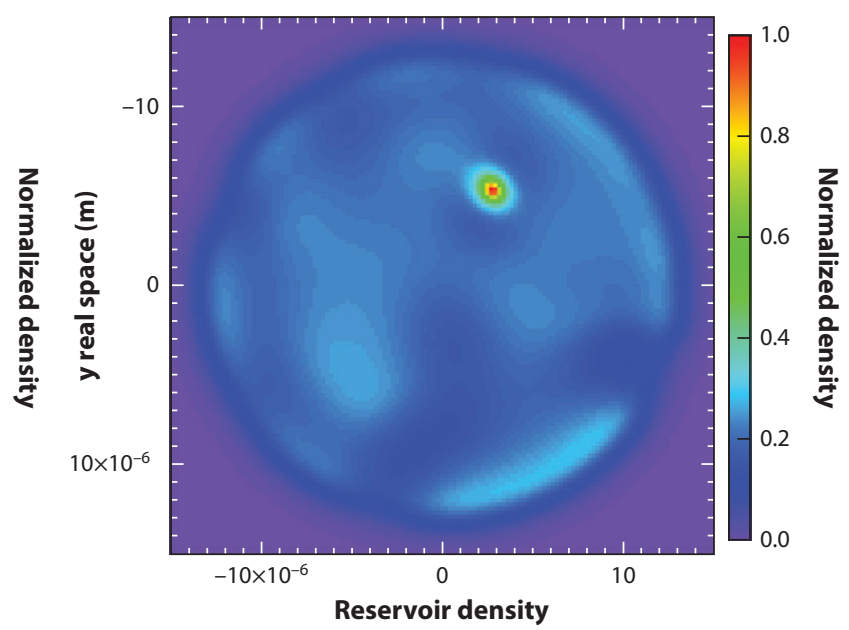

Figure 4

Theoretical density images of the lower polariton and the reservoir [from generalized Gross-Pitaevskii equation (GGPE)]. Excitation density is above the condensation threshold. Figure adapted from Reference 51. 


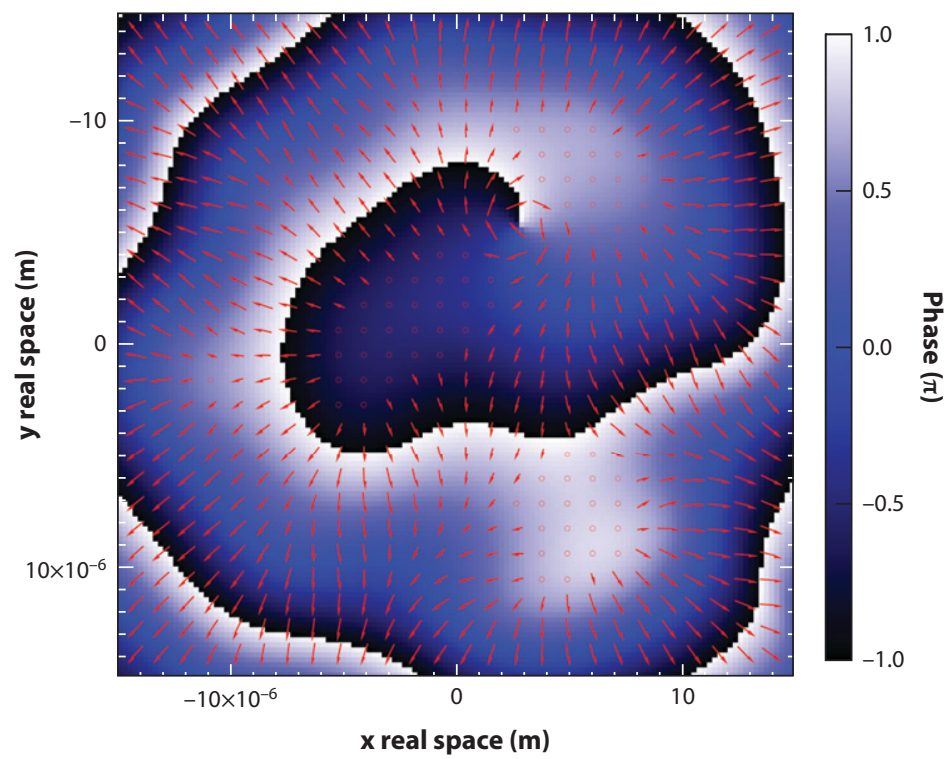

Figure 5

Phase structure of the condensate for the same parameters as described in Figure 4 . The $2 \pi$ phase shift at the vortex is clearly resolved, together with the smooth changes in the phase over the whole condensate, indicating that phase synchronization has been able to overcome the disorder of the sample. Of course, the phase is only defined within a global offset. Figure adapted from Reference 51.

$2 \pi$ phase shift when circumventing the vortex core. The smoothness of the variations of the phase across the whole of the condensate, despite the existence of a nonnegligible disorder, has been explained, both theoretically and experimentally, by the phase synchronization over the whole system (for example, see Reference 36 for the theoretical explanation and Reference 37 for the experiments).

Time-resolved experiments have also been realized in off-resonant excitation conditions. The precise pulse duration is not of major importance, provided it is shorter than the typical relaxation times. Typically, 100-fs pulses from a titanium-sapphire laser have been used. The detection is performed with a streak camera that allows resolution down to approximately 2 ps. Modeling such time-resolved experiments is somewhat more subtle. Indeed, as the reservoir is now not constantly replenished, the onset of stimulated scattering (a random process) at a given location immediately depletes the reservoir at the same position, not allowing a time-delayed growth at this point. This is due to our simplifying assumption that the whole population of the reservoir is connected to the lower polariton branch. This assumption is not physical in the case of time-resolved experiments. A delayed response of the population of reservoir excitons has to be included in the model. This is directly linked, as far as our understanding can tell, to the relaxation bottleneck.

A second reservoir of excitons is therefore included as the simplest way to describe the delayed coupling of a part of the large $k$ exciton population. The equation describing this second reservoir is the following:

$$
\frac{\partial n_{I}(r, t)}{\partial t}=P_{I}(r, t)-\gamma_{I} n_{I}(r, t)-\frac{1}{\tau_{I}} n_{I}(r, t) .
$$


Basically, large k polaritons, which cannot couple directly to the optically active polaritons at the center of the Brillouin zone, feed at a constant rate the reservoir of polaritons that are able to couple directly with $\mathrm{k}=0$ polaritons.

With such a simple model, many important observations can be reproduced, such as the delayed buildup of the population of polaritons in the lower branch after a nonresonant excitation (38). An amazing issue, experimentally observed in Reference 22 and reasonably well reproduced through the GGPE model that we just described, is the very rapid buildup of the long-range phase coherence in the condensate soon after the onset of stimulated scattering. In our experiments, as well as in the experiments of other groups (39), the onset of long-range coherence indeed appears within the experimental resolution, even for points that are 10-microns distant.

According to the resolution of the dynamics of GGPEs, with the introduction of an initial random phase in the lower polariton branch, we observe that, initially, the phase is strongly nonuniform with the presence of a multiplicity of vortices in the excitation spot. The buildup of a common phase appears to correspond to a mechanism very similar to the mechanism proposed by Kibble (40) and Zurek (41), meaning a combination of the movement of the core of the vortices toward the edge of the excitation spot together with the annihilation of counter-winding vortices (42). This annihilation of random vorticity indeed occurs in only a few picoseconds in the model for parameters that correspond as much as possible to the experimental configuration.

\section{PENROSE-ONSAGER CRITERION}

A polariton condensate is a somewhat unconventional condensate, making it interesting to test whether it would follow the very fundamental prescriptions of more standard Bose-Einstein condensates. One of the simplest predictions comes from Penrose \& Onsager, who introduced the Penrose-Onsager criterion (43). This criterion is based on the use of the single-particle density matrix $\rho\left(x, x^{\prime}\right)=\psi^{\dagger}(x) \psi\left(x^{\prime}\right)$ and simply states that Bose-Einstein condensation corresponds to the case in which the largest eigenstate value of this density matrix is of the order of the total number of particles in the system. An experimental measurement of the density matrix is a challenging task.

The density matrices shown in Figure 6 have been experimentally obtained through a tomographic procedure on a precise area of the sample where the condensate is confined to a onedimensional potential. This allows the tomographic procedure, where the stability of the interferometer has to be guaranteed over experimentally accessible times, to be experimentally feasible.

The results of the reconstruction of the density matrix are shown in Figure 5, and they indeed show that even in the presence of disorder and interaction between polaritons and despite the dissipative nature of polaritons, their distribution follows the criterion proposed by Penrose $\&$ Onsager (43). Below the condensation threshold, the occupancy of the states follows a standard thermal distribution, whereas above the threshold the macroscopic occupancy of a single level is clearly evidenced.

\subsection{Topological Defects: Specificities in a Spinor Condensate}

In the case of atomic condensates, one of the important steps in the study of the superfluidity of the condensate is the observation of quantized vortices. The idea consists of using the proper means to put the condensate in rotation and observing the arrangement of the vortex cores $(45,46)$. Although the rotation of the phase around the core of the vortex is not measured in the first experiments, the authors provided a beautiful illustration of the expected effects (47). In a further experiment, Wolfgang Kertterle's group managed to interfere with a rotating condensate possessing one vortex by using a nonrotating part of the same condensate. Interference fringes are 
a

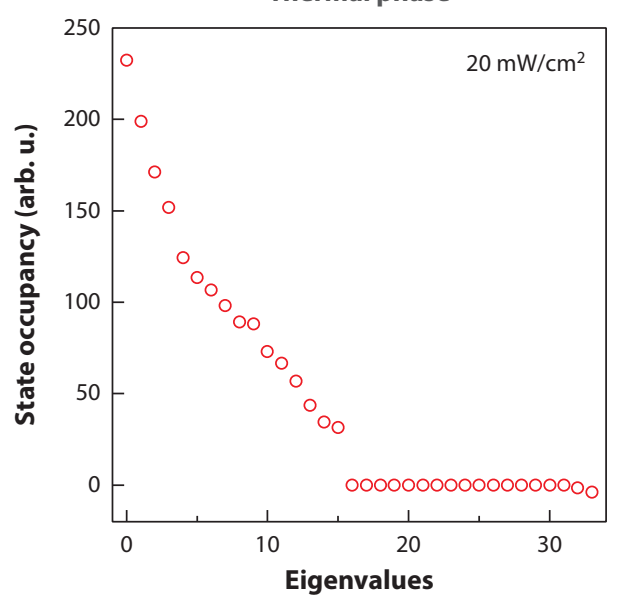

b

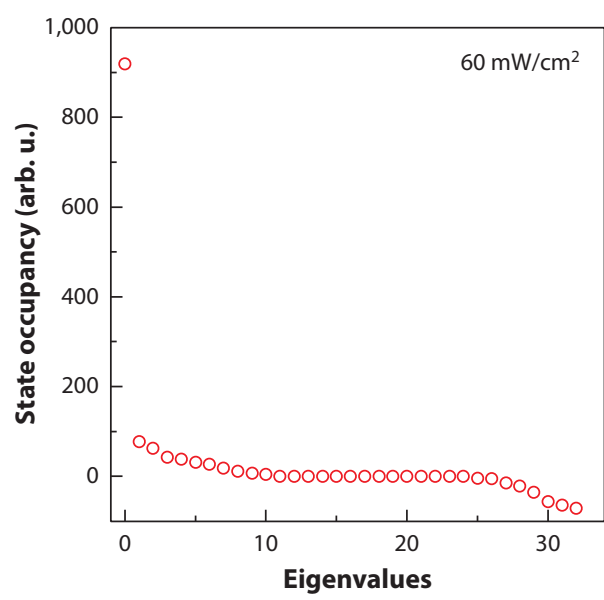

Figure 6

Eigenvalues of the density matrix of a one-dimensional (1D) condensate of polaritons, obtained from the reconstruction of the density matrix. (a) Below the condensation threshold. (b) Above the threshold at which the lowest eigenstate contains a macroscopic number of particles. Figure adapted from Reference 44.

clearly observed when a fork-like dislocation of the fringe pattern is viewed at the position of the vortex (48). Such a fork-like dislocation is outstanding evidence for the fact that the phase is changing by $2 \pi$ around the core of the vortex.

In the case of polariton condensates, the occurrence of fork-like dislocations, as seen in Figure 7, has been observed already in the first interference images (for example, see pages 116 and 118 in Reference 13 and page 96 in Reference 49). This observation has come as a surprise to us, as we did not proceed to any rotation of the condensate. With a detailed study, and with the support of the modeling that we briefly described in the previous paragraph, we have been able to understand that the occurrence of vortices was the double result of the dissipative nature of the condensate together with the presence of disorder in the sample (50).

Details of the interpretation of the creation of such vortices can be found in References 41 and 51. This interpretation also provides a detailed understanding of the dynamics of the creation of vortices after the excitation by a short pulse and allows us to explain why the movement of vortices can be resolved through time-resolved experiments in which the initial phase of the system is random and the experiment is performed by averaging millions of successive realizations (52). Indeed, under 100-fs pulse excitation, the buildup of the condensate occurs within approximately 30 ps (getting shorter as the excitation intensity is increased) and coherence over macroscopic distances appears within less than our time resolution of approximately 3 ps (38).

In fact, some of the trajectories of the randomly created vortices follow a path that is imposed by the shape of the disorder landscape, and the vortices are the results of the flow of polaritons in this disorder. At the same time, phase synchronization occurs (37) that allows stabilizing the phase of the whole population, despite the presence of disorder, within a very short delay demonstrated by direct measurements $(37,38)$.

Direct pumping of the polariton fluid, with a beam of appropriate winding, allows direct creation of a quantized vortex and even observation of its deterministic time evolution (53). In such experiments, a parametric amplification scheme is utilized that allows continuous feeding of the condensate without destroying the imprinted phase (54). 

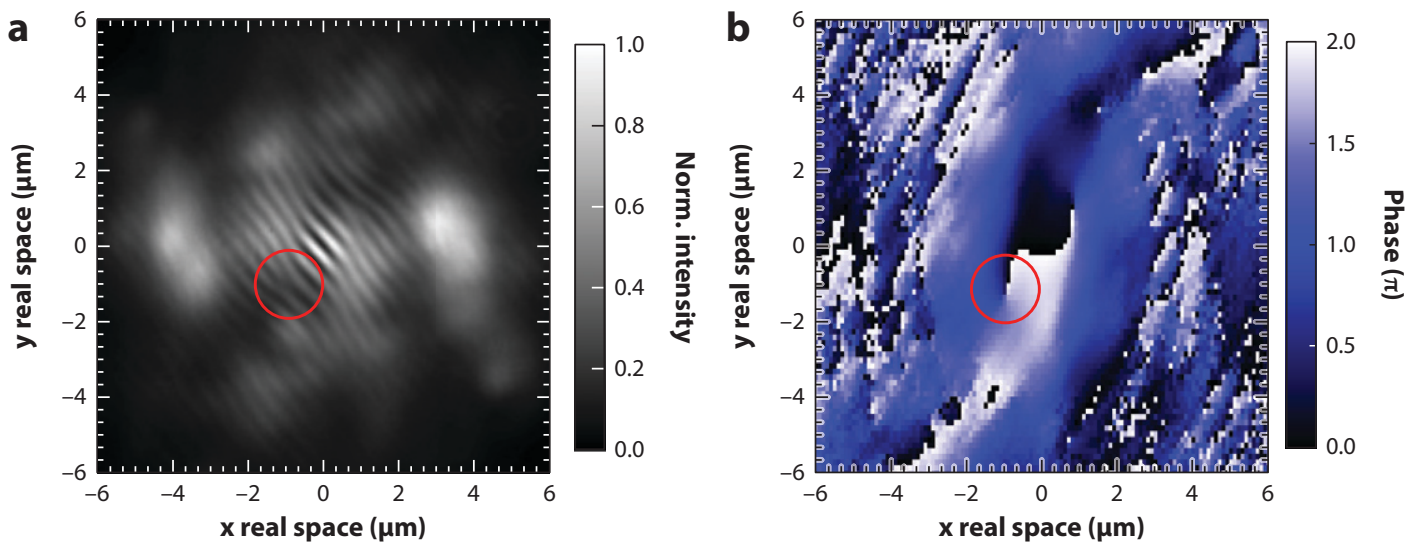

Figure 7

(a) Interference pattern, between the condensate and its centrosymmetric image, featuring a fork-like dislocation (see the position outlined by a red circle). Fringes give evidence for the long-range order. The fork-like dislocation highlighted by a circle corresponds to a quantized vortex. $(b)$ The Fourier transform of the pattern allows demonstrating a $2 \pi$ phase shift around the core of the vortex. Figure adapted from Reference 51. Abbreviation: Norm., normalized.

Following on the same track, it is important to point out that polaritons carry a spin. The spin of the polariton comes from the two possible circularly polarized states of its photon constituent and the spin of its exciton part. The spin of the exciton is built from the spin and angular momentum of the electron and hole states making the exciton (for example, details may be found in 55). Yuri Rubo (56) predicted in 2007 that the spinor properties of polaritons would allow, in principle, the observation of half vortices. For half vortices, the $2 \pi$ phase shift when circumventing the vortex core comes from a $\pi$ rotation of the polarization together with a $\pi$ phase shift (in the linear polarization basis).

When transforming into the circular polarization basis, a half vortex consists of a quantized vortex in one of the circular polarizations of the condensate and no vortex in the other one. This is exactly what the experiments carried out by Lagoudakis have evidenced (see Figure 8) (57). Once again, as for full-quantized vortices, the half vortices are created by the flow of polaritons in the disordered landscape imposed by the DBR mirrors of the cavity. Half vortices cannot be observed in superfluid $\mathrm{He}_{4}$ but are expected in spinor condensates such as $\mathrm{He}_{3}$ and cuprate superconductors $(58,59)$.

Then, following the arguments of Gregory Volovik (60), such half vortices should constitute the building blocks of the possible phase singularities in a polariton condensate. In particular, a full-quantized vortex corresponds to the superposition of two half vortices that have the same winding.

It has been possible to observe the dissociation of a full vortex into two half vortices through time-resolved interferometry measurements (see Figure 9 and Reference 61 for details). For these experiments, a portion of the sample with low disorder is selected where half vortices are observed in the CW regime. Once again, the stabilization of the path of the vortices is obtained because of the disorder present in the sample. However, we now need to explain the reason why a full vortex could dissociate into two half vortices. Indeed, this implies that the disorder felt by the $\sigma^{+}$and $\sigma^{-}$parts of the condensate is different. As a matter of fact, a nonhomogeneous birefringent field is expected to be created by the inhomogeneous stress present because of the disorder in the sample. 

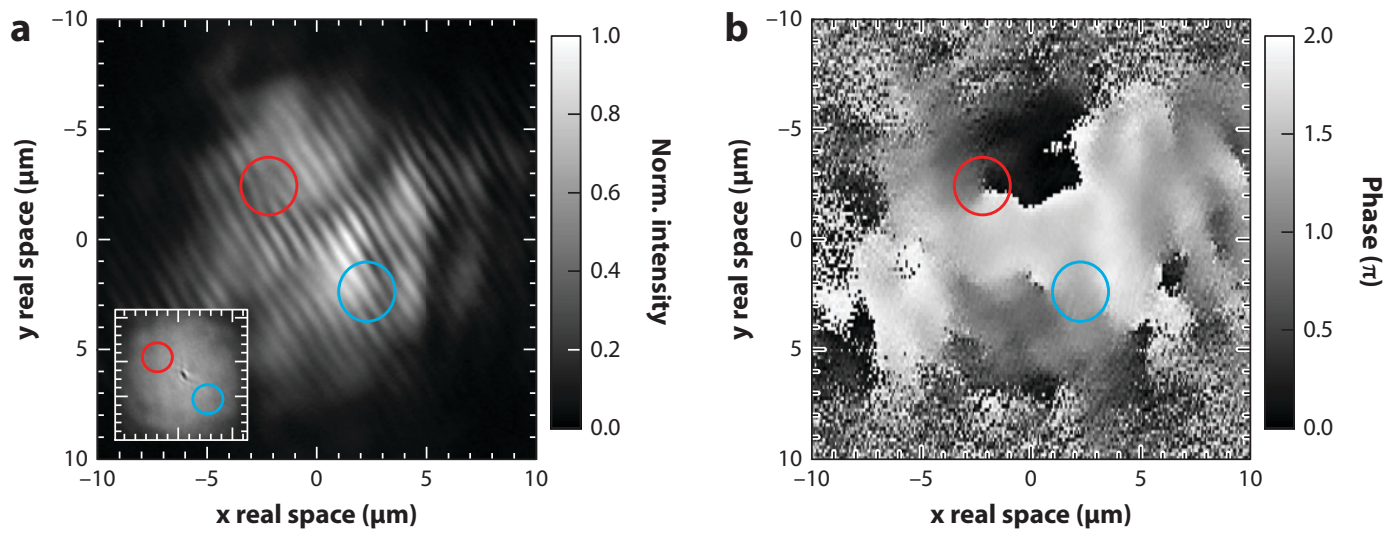

Figure 8

(a) Interferogram from the polarization mixing interferometer. We interfere the $\sigma^{+}$image with the centrosymmetric image of the $\sigma^{-}$ portion of the condensate. Although the symmetry should be the same as for the standard Michelson interferometer, the interferogram is clearly not centrosymmetric, as it features a fork-like dislocation on only one side (red circle) and no fork-like dislocation on the symmetric position (blue circle) with respect to the autocorrelation point. $(b)$ Phase extracted from the interference pattern. A phase singularity is clearly visible in the red circle, whereas a quasi-flat phase is seen in the symmetric position. Figure adapted from Reference 51.

Abbreviation: Norm., normalized.

Still following the predictions of Volovik (60), a careful study of the interference patterns while moving the excitation spot at the surface of the sample finds a pair of fork-like dislocations that does not fit what is observed in the case of a full vortex. Neither does it fit with a half-vortex pattern. The new fork structure (see Figure 10), upon interference of the $\sigma^{+}$and $\sigma^{-}$parts of the polariton condensate, shows two dislocations pointing in the same direction. This is clearly a case in which we observe the superposition of two half vortices in each circular polarization, with counter-circular windings of the phase. According to Volovik (60), this should allow for two configurations: a hyperbolic spin configuration in which the polarization of polaritons rotates counterclockwise when the observed position rotates clockwise around the core of the vortex and a configuration in which the polarization rotates clockwise, leading to a hedgehog vortex.

Measurement of the vortex structure in our sample evidences a clear rotation of the polarization of the polaritons (62). This polarization is plotted in the Poincaré sphere in Figure 11 and evidences a counterclockwise rotation when circumventing the core of the vortex. In a nonpolarized interferometer, the interference pattern does not show any fork-like dislocation.

This observation is typical of a hyperbolic vortex. The stability of the hyperbolic spin vortex, as observed experimentally, and not of the monopole spin vortex (or the hedgehog vortex), can be traced back to the difference in the interaction of the cores of the two half vortices that compose the spin vortex (see supplementary material of Reference 62).

\subsection{Polariton Superfluidity: How Polaritons and Light Can Share Common Properties}

The observation of phase defects in the polariton fluid points to the possibility that polaritons are superfluid. The demonstration of superfluidity is in fact rather simple if we follow the prescriptions of Wouters \& Carusotto (63). A polariton fluid is created through direct excitation in the polariton branch at a given angle. This gives rise to a polariton flow at a given speed (excitation at a given 

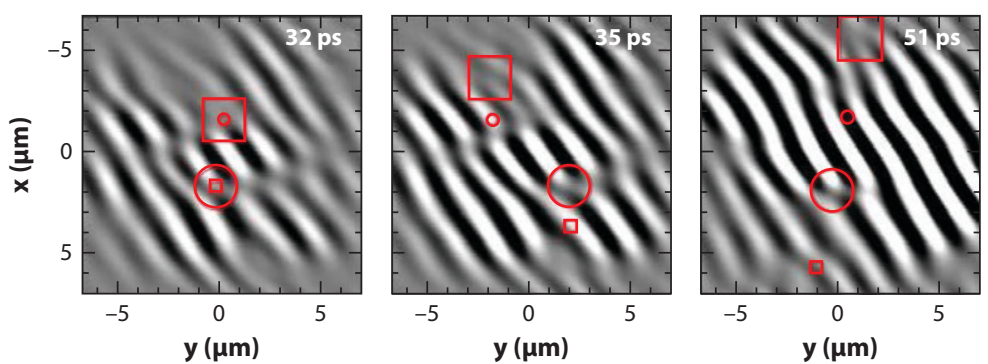

b
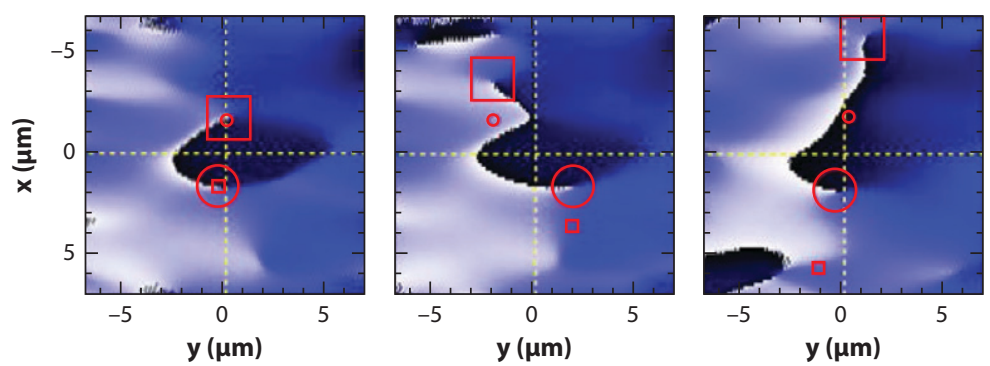

Figure 9

Snapshots of the time-resolved interferograms in a nonresonantly excited gas of polaritons. The top row shows the interferograms at different times and the lower row shows the time-resolved phase of the fluid. The condensate starts building up approximately 30 ps after the excitation pulse, and a full vortex is evidenced in the first frame by the two centrosymmetric dislocations. A few picoseconds later, the phase dislocations have evolved to noncentrosymmetric positions, showing that the full vortex has decomposed into two half vortices. Figure adapted from Reference 44.

position in the parabolic dispersion relation of polaritons means creating a wavepacket with well-defined energy and speed).

The presence of a defect then allows perturbation of the flow of the polariton fluid, and the turbulence is observed through Rayleigh scattering of the polaritons. The fluid may again be described by a GGPE, this time in the absence of a reservoir. We use GGPE with resonant excitation so that the phase of the excitation beam becomes imprinted in the condensate as given by the last term of the RHS:

$$
i \hbar \frac{\partial \psi(r, t)}{\partial t}=\left\{-\frac{\hbar^{2} \nabla^{2}}{2 m_{L P}}+V_{d}(r, t)-\frac{i \gamma \hbar}{2}+\hbar g|\psi(r, t)|^{2}\right\} \psi(r, t)+F_{p} e^{i\left(\omega_{p} t-k_{p} r\right)} .
$$

If the density is large enough that the speed of sound in the fluid is larger than the velocity of the polariton fluid, no turbulence should be observed.

The speed of sound in the fluid is given by $c_{s}=\sqrt{\frac{g|\psi|^{2}}{m}}$, when the speed of the flow $v_{\text {flow }}=\frac{\hbar k_{\|}}{m}$ is directly imposed by the angle of the exciting laser.

The first series of experiments in the direction have been performed by Amo and coworkers $(64,65)$ by using an optical parametric amplification configuration and sending a flow of polaritons with a given speed toward an obstacle. The experiment provides ample evidence of the transition to a superfluid regime when the density of the fluid is large enough to allow the 

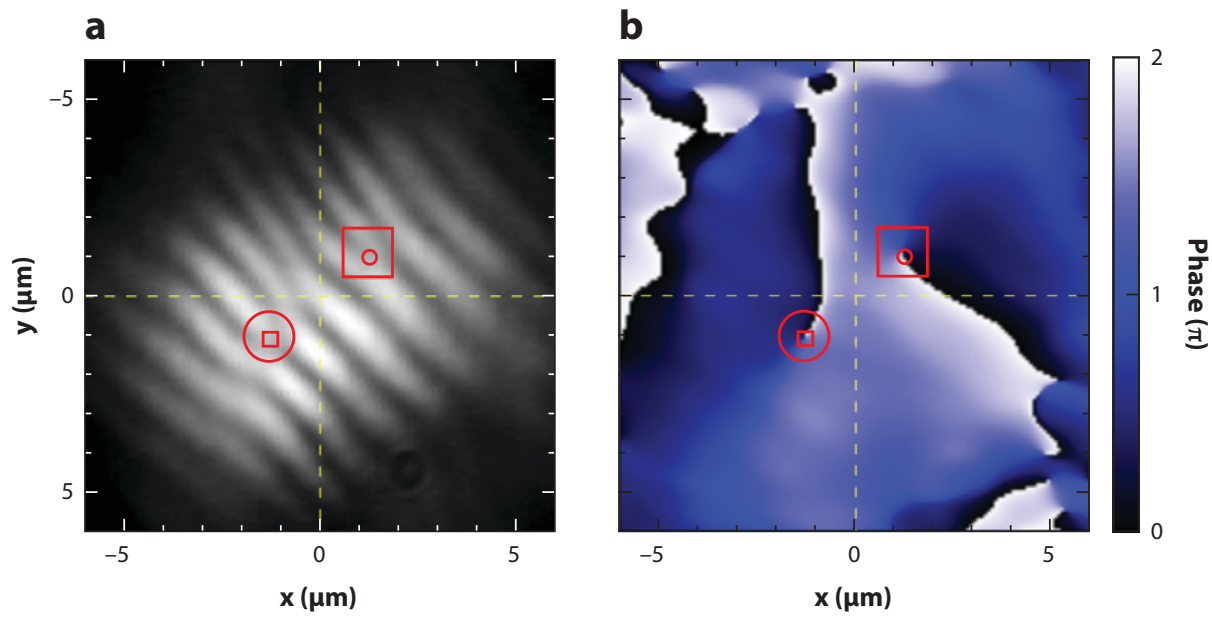

Figure 10

(a) Interferogram from the polarization mixing interferometer. We interfere with the $\sigma^{+}$image using the centrosymmetric image of the $\sigma^{-}$portion of the condensate. Although the symmetry should be the same as for the standard Michelson interferometer, the interferogram is clearly not centrosymmetric, as it features two fork-like dislocations pointing in the same direction. This is an indication for two half vortices of counterwindings. (b) Phase extracted from the interference pattern. The two-phase singularities indeed have opposite windings. Figure adapted from Reference 44.

speed of sound to overcome the speed of the polariton flow. In contrast, when the speed of the fluid is too large compared with the speed of sound, Cherenkov waves are observed around the obstacle.

Nardin and coworkers $(66,67)$ have extended the principle of such measurements to the time domain by carrying out homodyne detection measurements on the flow of polaritons (see also 68). The principles of the experiment are sketched in Figure 12. Time-resolved phase and amplitude of a fluid of polaritons can be retrieved through homodyne mixing with a delayed pulse from the laser (see Figure 13). The time resolution is given by the pulse energy resolution of approximately $1 \mathrm{meV}$ and 1 ps.

The experiment retrieves both the phase and the amplitude of the flow of polaritons around an obstacle. The regimes of no turbulence in the case of a large-enough density of the polariton fluid and of Cherenkov waves in the case of a too-small density of the fluid compared with the speed of polaritons are observed.

The advantage of the heterodyne technique is that the phase of the fluid, although imposed initially by the excitation laser, is free to evolve after the end of the pulse. Also, the measurement of the phase allows us to see very clearly the presence of quantized vortices, once again corresponding to fork-like dislocations in the interference pattern on the CCD camera. The measurement of the phase also allows access to the local speed of the polariton fluid as a function of position and time around the obstacle.

The theoretical analysis of the predictions of GGPEs showed that, as has been observed in atom condensates, dark solitons should form, depending on the precise excitation conditions. Following such predictions [for example, see the work of Pigeon et al. (70)], several experimental works have been published demonstrating the existence of such dark solitons in the case of polariton fluids $(71,72)$. Once again, as polaritons carry a spin, it has also been possible to study the peculiarities of such dark solitons for spinor fluids, namely the emergence of half solitons (73). Similar to half vortices, half solitons appear in one of the helicities of the fluid 
a

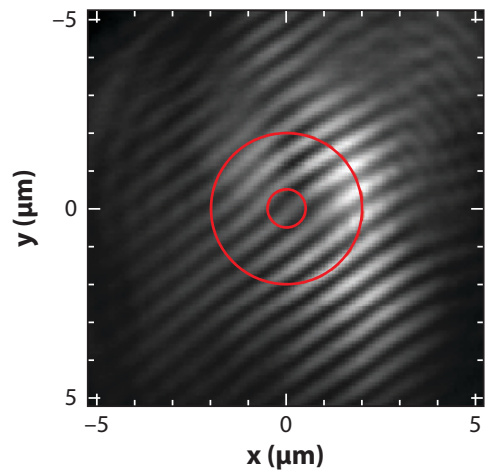

b

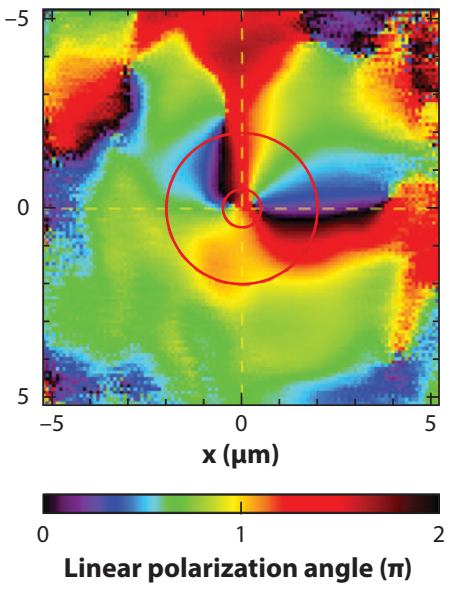

Figure 11

(a) Interferogram at the position of the hyperbolic spin vortex. No fork-like dislocation is observed. $(b)$ Changes in the polarization vector around the core of the vortex (the angle is plotted in the Poincaré sphere). Figure adapted from Reference 44.

and not in the other (74). Experiments using heterodyne detection have been able to resolve the dynamical behavior of dark solitons and, in particular, their stability and their dissociation into vortex streets $(72,75)$.

\section{BOGOLIUBOV DISPERSION}

One of the distinctive outstanding ideas in the theory of superfluidity comes from the seminal work of Bogoliubov (76). According to the theory that he has developed, the elementary excitations of the superfluid phase of weakly interacting bosons, such as polaritons, consist in a superposition of a propagating particle and its counter-propagating counterpart, an absence of particle or a hole. In the superfluid limit, both branches tend to linearize: the normal branch of particles at positive energies and the so-called ghost branch of holes at negative energies (77). Although excitations in superfluids have been studied quite extensively, particularly in superfluid helium first $(78,79)$ and ultra-cold atom vapors $(80,81)$, the first experimental evidence for the Bogoliubov transformation was published quite recently by the Ketterle group (82). In these experiments, Vogels et al. (82) probe the presence of the negative energy branch through the diffraction of a sound wave that is moved onto the atom condensate.

The experiments that my research group has been performing on the polariton Bogoliubov transformation are based on the ideas developed by Michiel Wouters \& Iacopo Carusotto (83). Our experiments have been carried out after some attempts to observe the Bogoliubov transformation using luminescence (84) where no evidence was found for the ghost branch. The idea of Reference 61 is to probe the dispersion of the polariton fluid excitations through four-wave mixing (FWM) experiments. In these FWM experiments, a superfluid of polaritons is created by a $\mathrm{k}=0$ pump beam, and the properties of the system are probed through the diffraction of a probe beam sent at an angle, therefore probing the dispersion of the fluid. The experiment has been described in References 85 and 86, and the results (see Figure 14) show clearly the existence 


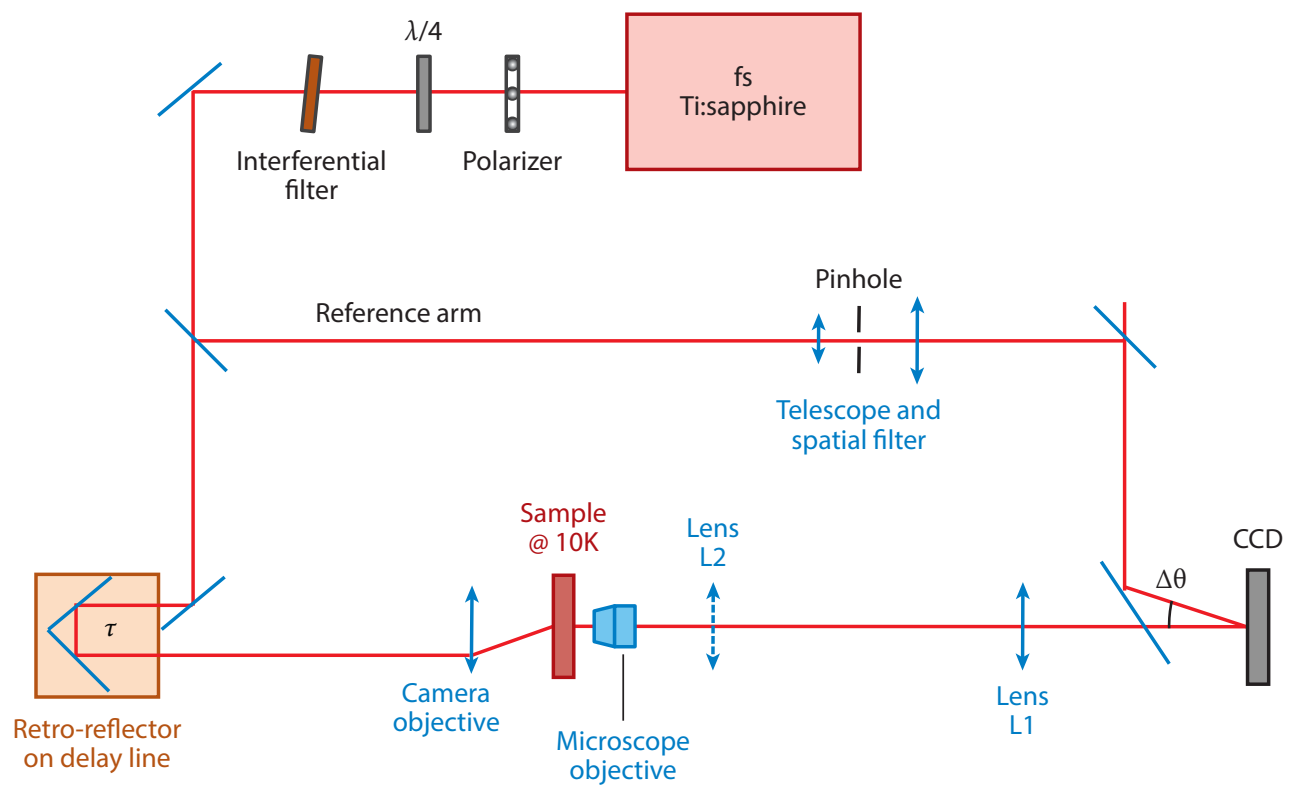

Figure 12

Schematics of the homodyne system. Pulses from a Ti:sapphire laser are split into two arms. One excites the sample at a given angle, given a well-defined speed to the polariton fluid. The light emitted by the sample is interfered with by the second pulse from the laser. The interference fringes are then Fourier transformed so as to obtain the time-resolved density and phase of the fluid. Figure adapted from Reference 69. Abbreviation: CCD, charge-coupled device; fs, femtosecond

of two branches instead of one: one branch at positive energies and one at negative energies (see Figure $14 a$ ). Figure $14 b$ also evidences the linearization of the dispersion relation of the polaritons and the appearance of a ghost branch at negative energies, above the threshold for superfluidity of the polariton gas.

In the case of the FWM experiment, once again we use GGPEs to model the behavior of the condensate. As we are using resonant excitation, a reservoir of excitons is not created and is not used in the modeling. However, the description of the Bogoliubov transformation requires the use of a properly adapted wavefunction that deals with the excitations of the system. The wavefunction reads as follows:

$$
\psi(r, t)=\varphi_{o}(r, t)+u(r, t) e^{i k r}+v^{*}(r, t) e^{-i k r},
$$

where $\varphi_{o}(r, t)$ is the $\mathrm{k}=0$ coherent polariton fluid created by the pump pulse, and $u$ and $v$ are the counter-propagating excitations of the system. The GGPE can be numerically solved in order to obtain the intensity and the energies of the two branches. At large-enough densities, the ghost branch shows the same intensity as the normal branch. The position of the resonances are reproduced in Figure $\mathbf{1 1}$ and fit quite well with the observed spectrum. In particular, the fact that the ghost branch does not appear linear in our measurements is well reproduced by taking into account the finite lifetime of the polaritons. 


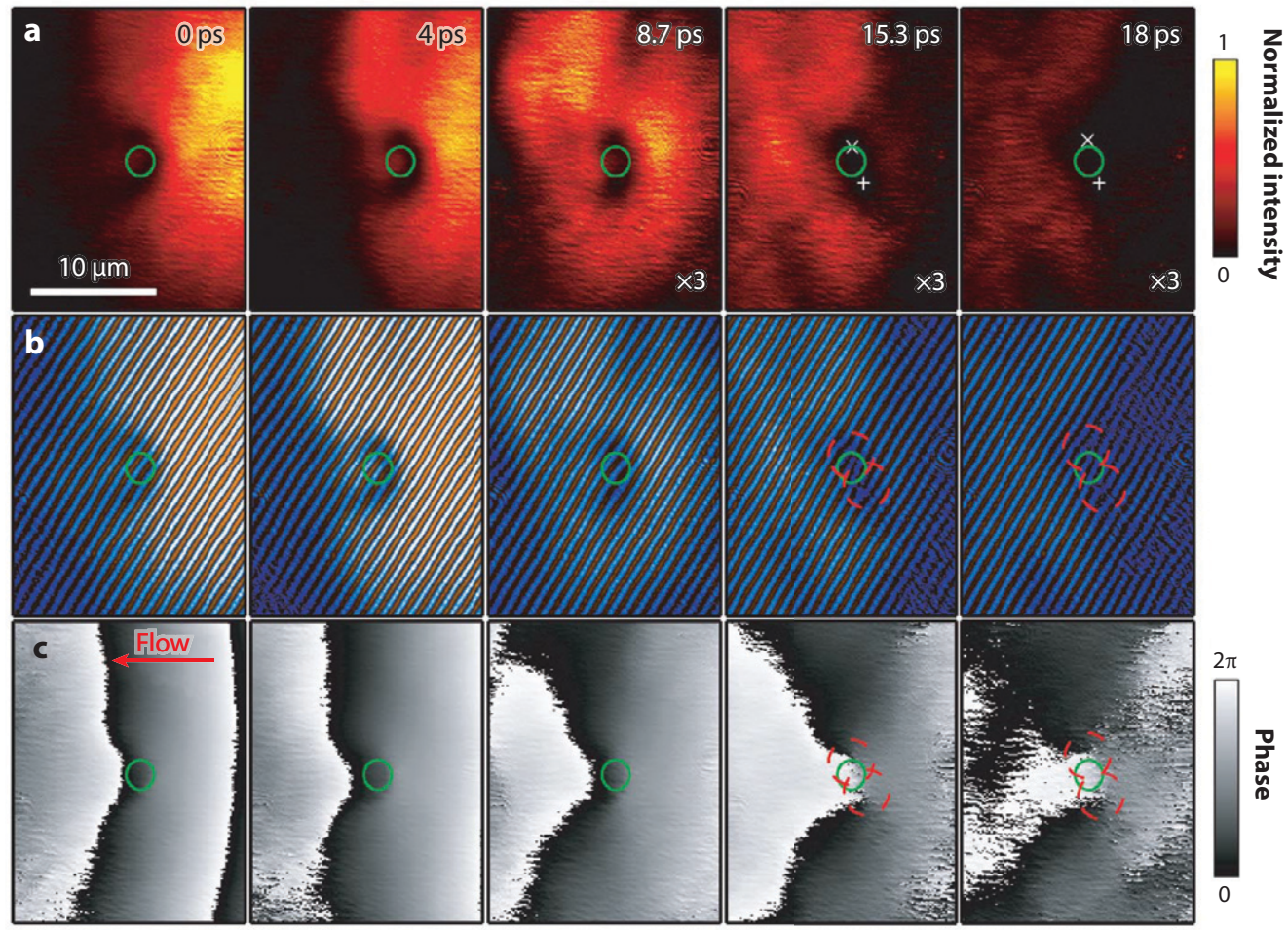

Figure 13

Superfluid behavior without turbulence. The three rows respectively show at different delays $(a)$ the polariton density, $(b)$ the fringes of the measured interferogram in a saturated color scale, and $(c)$ the polariton phase. The estimated obstacle position is indicated with a green circle, and the polariton flow goes leftward (see the red arrow in the third row). At low speed, the wavepacket passes the obstacle without any turbulence. When the polariton population has significantly decayed (last two columns), a vortex pair is created at the boundary of the obstacle. The pair remains stitched to the defect for several picoseconds and disappears in the noise when the polariton population has decayed. For the sake of visibility, the density scale has been multiplied by three for the last three panels of the first line. Figure adapted from Reference 69.

\section{CONCLUSIONS}

I hope that I have been able to convince the reader that there is an amazing richness in the field of microcavity exciton-polaritons. The ease with which the internal properties of the condensate can be assessed thanks to the photonic portion of the polariton that leaks out of the cavity allows a wide range of measurements that would not otherwise be possible. In particular, interferometric measurements allow a very didactic visualization of the phase structure of a polariton fluid, showing a wide range of phase defects. Although very similar properties have been observed for polariton condensates as those found in cold atom condensates, significant differences are evidenced because of the specific nature of polaritons. Such differences come from the driven dissipative nature of the condensate, with a very short lifetime for polaritons, the relatively strong interactions between the quasiparticles, and the presence of disorder due to the solid-state environment of the system. Many unsolved issues still exist in the understanding of the details of the interactions between polaritons. It is clear that the current models are too simple to describe the complexity of such interactions. The use of polariton Feshbach resonance, recently evidenced for polariton, might bring interesting features (87). 

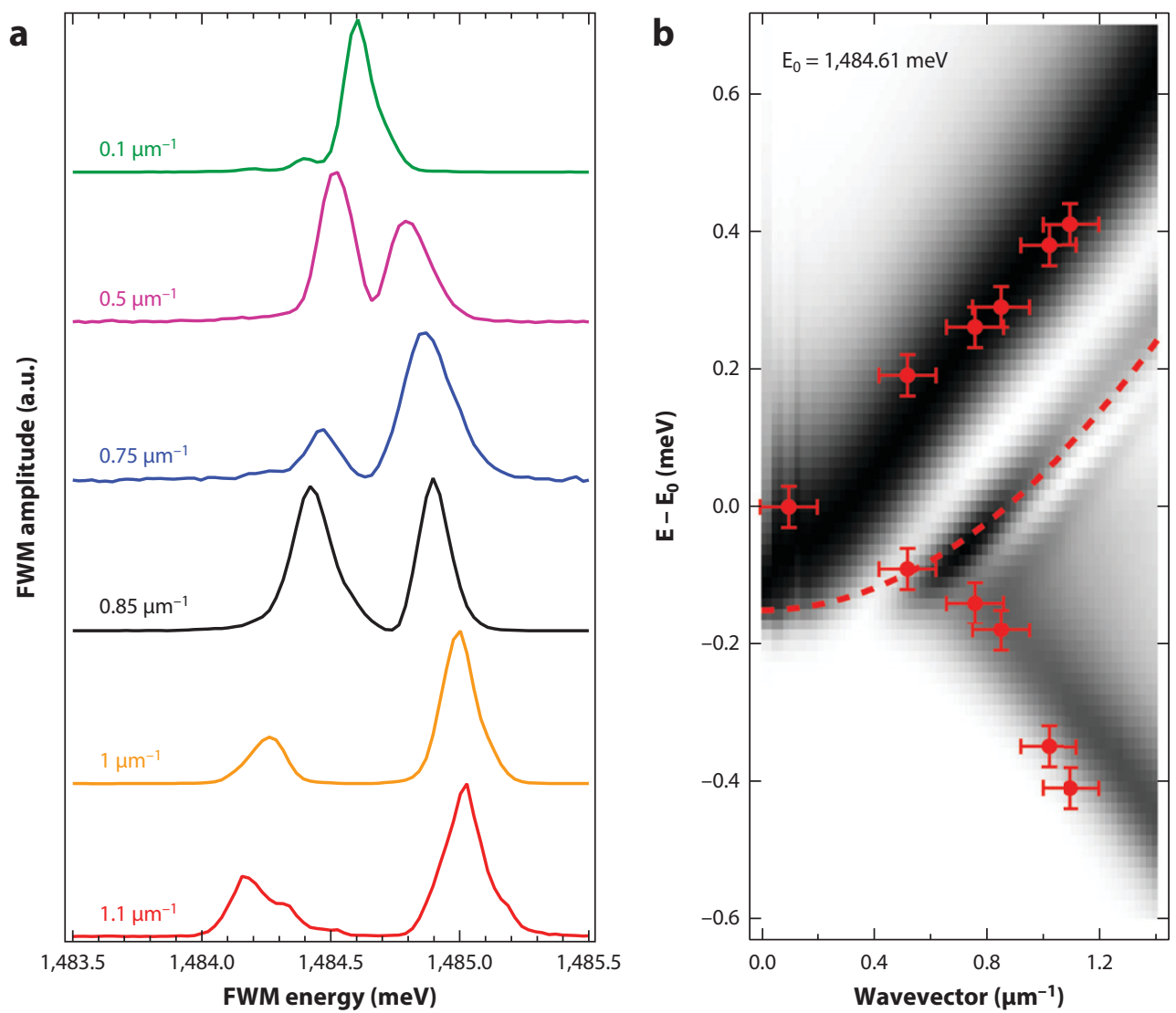

Figure 14

(a) Four-wave mixing (FWM) signals at a given density for different wavevectors of the probe. (b) Polariton dispersion relation. The experimental peaks in red are compared with the dispersion in the absence of pumping (dashed red) and with the results of our model that takes into account the finite lifetime of the polariton fluid. A clear linearization of the dispersion, together with the appearance of a ghost branch, is observed. Figure reproduced from Reference 85.

\section{DISCLOSURE STATEMENT}

The author is not aware of any affiliations, memberships, funding, or financial holdings that might be perceived as affecting the objectivity of this review.

\section{ACKNOWLEDGMENTS}

This work is inspired by successive works in my group (but not only by my group, as I integrate in this review the works of Pascale Senellart, Maxime Richard, Jacek Kasprzak, Alberto Amo, and others), over the past 15 years, starting with that of Michele Saba, when we first envisaged the possibility of condensation of polaritons and designed an original way to produce efficient trapping of polaritons. This work has been followed by what I call the dream team of LOEQ with, in order of appearance on stage, Stefan Kundermann, Reda Idrissi Kaitouni, Ounsi El Daif, Konstantinos Lagoudakis, Taofiq Paraïso, Gael Nardin, Roland Cerna, Verena Kohnle, Francesco Manni, and Gabriele Grosso. It has also been supported very strongly by a group of extremely talented postdocs that I wish to congratulate warmly for their outstanding work: Maxime Richard, 
Augustin Baas, Thierry Guillet, Barbara Pietka, Yoan Léger and, last but not least, Stéphane Trébaol. I also would like to convey my most sincere thanks to the theoreticians who allowed us to understand our results by spending enough time with us and by putting into simple words the results of their equations. In particular, Cristiano Ciuti, Vincenzo Savona, Michiel Wouters, Tim Liew, Alexei Kavokin, and Yuri Rubo deserve very special acknowledgments. Antonio Quattropani and Paolo Schwendiman have constantly helped with many discussions and their indefectible support of theorist-experimentalist collaborations. Last, but not least, this work would not have been possible without the very close collaboration with the Dang group in Grenoble and, in particular, without the very high quality samples prepared by Régis André. The work has been carried out within the framework of the Quantum Photonics National Center of Competence in research financed by the Swiss National Science Foundation. It has also been financed by the European Research Council under the Polaritronics Advanced Grant. Complementary funding for conferences and meetings has been obtained through the Latsis Foundation and the Polatom network of the European Science Foundation.

\section{LITERATURE CITED}

1. Blatt JM, Böer KW, Brandt W. 1962. Phys. Rev. 126:1691-92

2. Moskalenko SA. 1962. Sov. Phys. Solid State 4:199-204

3. Anderson MH, Ensher JR, Matthews MR, Wieman CE, Cornell EA. 1995. Science 269:198-201

4. Davis KB, Mewes MO, Andrews MR, van Druten NJ, Durfee DS, et al. 1995. Phys. Rev. Lett. 75:3969-72

5. Keldysh LV, Kozlov AN. 1968. J. Exp. Theor. Phys. USSR 27:521-28

6. Wertz E, Ferrier L, Solnyshkov DD, Johne R, Sanvitto D, et al. 2010. Nat. Phys. 24:4502

7. Sturm C, Tanese D, Nguyen HS, Flayac H, Galopin E, et al. 2014. Nat. Commun. 5:3278

8. Hulin D, Mysyrowicz A, Benoît à la Guillaume C. 1980. Phys. Rev. Lett. 45:1970-73

9. Lin JL, Wolfe JP. 1993. Phys. Rev. Lett. 71:1222-25

10. Eisenstein JP, MacDonald AH. 2004. Nature 432:691-94

11. Koyama F. 2006. J. Lightwave Technol. 24:4502-13

12. Jewell JL, Harbison JP, Scherer A, Lee YH, Florez LT. 1991. IEEE J. Quantum Electron. 27:1332-46

13. Kasprzak J. 2006. Condensation of exciton polaritons. PhD Thesis, Univ. Joseph Fourier, Grenoble

14. Nelsen B, Liu G, Steger M, Snoke DW, Balili R, et al. 2013. Phys. Rev. X 3:041015

15. Richard M. 2004. Quasi-condensation de polaritons sous excitation incohérente dans les microcavités II-VI à base de CdTe. PhD Thesis, Univ. Joseph Fourier, Grenoble

16. Savona V, Hradil Z, Quattropani A, Schwendimann P. 1994. Phys. Rev. B 49:8774-79

17. Savasta S, Girlanda R. 1996. Phys. Rev. A 53:2716-26

18. Kasprzak J, Richard M, Baas A, Deveaud B, André R, et al. 2008. Phys. Rev. Lett. 100:067402

19. Tempel J-S, Veit F, Aßmann M, Kreilkamp LE, Rahimi-Iman A, et al. 2012. Phys. Rev. B 85:075318

20. Kasprzak J, Richard M, Kundermann S, Baas A, Jeambrun P, et al. 2006. Nature 443:409-14

21. Dang LS, Heger D, André R, Boeuf F, Romestain R. 1998. Phys. Rev. Lett. 81:3920-23

22. Senellart P, Bloch J. 1999. Phys. Rev. Lett. 82:1233-36

23. Balili R, Hartwell V, Snoke D, Pfeiffer L, West K. 2007. Science 316:1007-10

24. Bajoni D, Senellart P, Wertz E, Sagnes I, Miard A, et al. 2008. Phys. Rev. Lett. 100:047401

25. Christopoulos S, Baldassarri Höger von Högersthal G, Grundy AJD, Lagoudakis PG, Kavokin AV, et al. 2007. Phys. Rev. Lett. 98:126405

26. Li F, Orosz L, Kamoun O, Bouchoule S, Brimont C, et al. 2013. Phys. Rev. Lett. 110:196406

27. Daskalakis KS, Maier SA, Murray R, Kéna-Cohen S. 2014. Nat. Mater. 13:271-78

28. Plumhof JD, Stöferle T, Lijian Mai U. 2014. Nat. Mater. 13:242-51

29. Bajoni D. 2012. J. Phys. D 45:313001

30. Imamoglu A, Ram RJ, Pau S, Yamamoto Y. 1996. Phys. Rev. A 53:4250-53

31. Szymanska MH, Keeling J, Littlewood PB. 2006. Phys. Rev. Lett. 96:230602 
32. Keeling J, Berloff NG. 2008. Phys. Rev. Lett. 100:250401

33. Wouters M, Carusotto I. 2007. Phys. Rev. Lett. 99:140402

34. Tassone F, Piermarocchi C, Savona V, Quattropani A, Schwendimann P. 1997. Phys. Rev. B 56:7554-63

35. André R, Heger D, Le Si Dang Y. 1998. J. Cryst. Growth 184/185:758-62

36. Wouters M. 2008. Phys. Rev. B 77:121302

37. Baas A, Lagoudakis KG, Richard M, André R. 2008. Phys. Rev. Lett. 100:170401

38. Nardin G, Lagoudakis KG, Wouters M, Richard M, Baas A, et al. 2009. Phys. Rev. Lett. 103:256402

39. del Valle E, Sanvitto D, Amo A, Laussy FP, André R, et al. 2009. Phys. Rev. Lett. 103:096404

40. Zurek WH. 1984. Nature 317:505-8

41. Kibble TBH. 1976. J. Phys. A 9:1387-98

42. Kagan Y, Svistunov BV, Shlyapkinov GV. 1985. JETP Lett. 42:209-12

43. Penrose O, Onsager L. 1956. Phys. Rev. 104:576-84

44. Manni F. 2013. Coherence and topological defects in exciton-polariton condensates. PhD Thesis, EPFL, Lausanne

45. Matthews MR, Anderson BP, Haljan PC, Hall DS, Wieman CE, Cornell EA. 1999. Phys. Rev. Lett. 83:2498-501

46. Madison KW, Chevy F, Wohlleben W, Dalibard J. 2000. Phys. Rev. Lett. 84:806-9

47. Abo-Shaeer JR, Raman C, Vogels JM, Ketterle W. 2001. Science 292:476-79

48. Inouye S, Gupta S, Rosenband T, Chikkatur AP, Görlitz A, et al. 2001. Phys. Rev. Lett. 87:080402

49. Kundermann S. 2006. Coherence properties of microcavity polaritons: from parametric scattering to Bose-Einstein condensation. PhD thesis, EPFL, Lausanne

50. Lagoudakis KG, Wouters M, Richard M, Baas A, Carusotto I, et al. 2008. Nat. Phys. 4:706-10

51. Lagoudakis KG. 2011. On the Physics of Exciton-Polariton Condensates. Lausanne, Switz.: EPFL Press

52. Lagoudakis KG, Manni F, Pietka B, Wouters M, Liew TCH, et al. 2011. Phys. Rev. Lett. 106:115301

53. Sanvitto D, Marchetti FM, Szymanska MH, Tosi G, Baudisch M, et al. 2010. Nat. Phys. 6:527-33

54. Tosi G, Marchetti FM, Sanvitto D, Anton C, Szymanska MH, et al. 2011. Phys. Rev. Lett. 107:036401

55. Hermann C, Lampel G, Safarov VI. 1985. Ann. Phys. 10(6):22

56. Rubo YG. 2007. Phys. Rev. Lett. 99:106401

57. Lagoudakis KG, Ostatnick T, Kavokin AV, Rubo YG, André R, Deveaud-Plédran B. 2009. Science 326:974-76

58. Yamashita M, Izumina K, Matsubara A, Sasaki Y, Ishikawa O, et al. 2008. Phys. Rev. Lett. 101:025302

59. Kirtley JR, Tsuei CC, Martin Rupp JZ. 1996. Phys. Rev. Lett. 76:1336-39

60. Volovik GE. 2000. Proc. Natl. Acad. Sci. USA 97:2431-36

61. Manni F, Lagoudakis KG, Liew TCH, André R, Savona V, Deveaud B. 2012. Nat. Commun. 3:1309

62. Manni F, Léger Y, Rubo YG, André R, Deveaud B. 2013. Nat. Commun. 4:2590

63. Wouters M, Carusotto I. 2010. Phys. Rev. Lett. 105:020602

64. Amo A, Sanvitto D, Laussy FP, Ballarini D, del Valle E, et al. 2009. Nature 457:291-95

65. Amo A, Lefrère J, Pigeon S, Adrados C, Ciuti C, et al. 2009. Nat. Phys. 5:805-10

66. Nardin G, Grosso G, Leger Y, Pietka B, Morier-Genoud F, Deveaud-Plédran B. 2011. Nat. Phys. 7:635-41

67. Deveaud B, Nardin G, Grosso G, Léger Y. 2013. In Physics of Quantum Fluids: New Trends and Hot Topics in Atomic and Polariton Condensates, Vol. 177, ed. A. Bramati, M. Modugno, p. 177-200. New York: Springer

68. Sanvitto D, Pigeon S, Amo A, Ballarini D, De Giorgi M, et al. 2011. Nat. Photonics 5:610-14

69. Nardin G. 2011. Phase-resolved imaging of exciton polaritons. PhD Thesis, EPFL, Lausanne

70. Pigeon S, Carusotto I, Ciuti C. 2011. Phys. Rev. B 83:144513

71. Amo A, Pigeon S, Sanvitto D, Sala VG, Hivet R, et al. 2011. Science 332:1167

72. Grosso G, Nardin G, Léger Y, Morier-Genoud F, Deveaud-Plédran B. 2011. Phys. Rev. Lett. 107:245301

73. Flayac H, Shelykh IA, Solnyshkov DD, Malpuech G. 2010. Phys. Rev. B 81:045318

74. Hivet R, Flayac H, Solnyskhov DD, Tanese D, Boulier T, et al. 2012. Nat. Phys. 8:724-28

75. Grosso G, Nardin G, Morier-Genoud F, Léger Y, Deveaud-Plédran B. 2012. Phys. Rev. B 86:020509

76. Bogoliubov NN. 1947. J. Exp. Theor. Phys. USSR 11:23

77. Pitaevskii L, Stringari S. 2003. Bose-Einstein Condensation. Oxford, UK: Clarendon 
78. Jackson HW, Feenberg E. 1962. Rev. Mod. Phys. 34:68

79. Woods ADB, Cowley RA. 1973. Rep. Prog. Phys. 36:1135

80. Steinhauer J, Ozeri R, Katz N, Davidson N. 2002. Phys. Rev. Lett. 88:120407

81. Ozeri R, Katz N, Steinhauer J, Davidson N. 2005. Rev. Mod. Phys. 77:187

82. Vogels JM, Xu K, Raman C, Abo-Shaeer JR, Ketterle W. 2002. Phys. Rev. Lett. 88:060402

83. Wouters M, Carrusoto I. 2009. Phys. Rev. B 79:125311

84. Utsunomiya S, Tian L, Roumpos G, Lai CW, Kumada N, et al. 2008. Nat. Phys. 4:700

85. Kohnle V, Léger Y, Wouters M, Richard M, Portela-Oberli MT, Deveaud-Plédran B. 2011. Phys. Rev. Lett. 106:255302

86. Kohnle V, Leger Y, Wouters M, Richard M, Portella-Oberli MT, Deveaud B. 2012. Phys. Rev. B 86:064508

87. Takemura N, Trebaol S, Wouters M, Portella-Oberli MT, Deveaud B. 2014. Nat. Phys. 10:500 


\section{Contents}

Innovations in Statistical Physics

Leo P. Kadanoff ............................. 1

Many-Body Localization and Thermalization in Quantum Statistical Mechanics

Rahul Nandkishore and David A. Huse . . . . . . . . . . . . . . 15

Composite Fermion Theory of Exotic Fractional Quantum Hall Effect Jainendra K. Jain . . . . . . . . . . . . . . . . . . . . . 39

The Statistical Physics of Athermal Materials

Dapeng Bi, Silke Henkes, Karen E. Daniels, and Bulbul Chakraborty . . . 63

Napoleon Is in Equilibrium

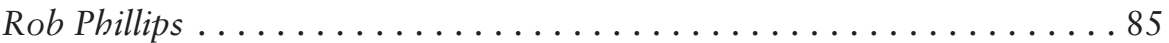

Assembly of Biological Nanostructures: Isotropic and Liquid Crystalline Phases of Neurofilament Hydrogels Cyrus R. Safinya, Joanna Deek, Roy Beck, Jayna B. Jones, and Youli Li . . . . . . . . . . . . . . . . . . . . . . . . . . . . . 113

Plutonium-Based Heavy-Fermion Systems

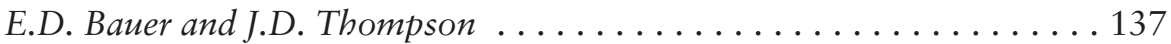

Exciton-Polariton Bose-Einstein Condensates Benoît Deveaud ................................ 155

Marginal Stability in Structural, Spin, and Electron Glasses Markus Müller and Matthieu Wyart ....................... 177

Ultracold Atoms Out of Equilibrium Tim Langen, Remi Geiger, and Jörg Schmiedmayer . . . . . . . . . . . 201

Motility-Induced Phase Separation Michael E. Cates and Julien Tailleur 
Physics of Viral Shells

Robijn F. Bruinsma and William S. Klug . . . . . . . . . . . . . . . 245

Amplitude/Higgs Modes in Condensed Matter Physics

David Pekker and C.M. Varma . . . . . . . . . . . . . . . . . . . . . . 269

Symmetry-Protected Topological Phases of Quantum Matter

T. Senthil . . . . . . . . . . . . . . . . . . . . . . . . . . 299

Spatial Localization in Dissipative Systems

E. Knobloch . . . . . . . . . . . . . . . . . . . . . . . . . . 325

Topological Crystalline Insulators and Topological Superconductors:

From Concepts to Materials

Yoichi Ando and Liang Fu . . . . . . . . . . . . . . . . . . 361

Universal Dynamics and Renormalization in Many-Body-Localized Systems Ehud Altman and Ronen Vosk . . . . . . . . . . . . . . . . . 383

Quantum Oscillations in Hole-Doped Cuprates

Suchitra E. Sebastian and Cyril Proust . . . . . . . . . . . . . 411

\section{Errata}

An online log of corrections to Annual Review of Condensed Matter Physics articles may be found at http://www.annualreviews.org/errata/conmatphys 